\title{
Optical studies of the nematic phase of an oxazole-derived bent-core liquid crystal
}

\author{
J. A. Olivares, ${ }^{1}$ S. Stojadinovic, ${ }^{2}$ T. Dingemans, ${ }^{3}$ S. Sprunt, ${ }^{2}$ and A. Jáklii ${ }^{1}$ \\ ${ }^{1}$ Liquid Crystal Institute, Kent State University, Kent, Ohio 44242, USA \\ ${ }^{2}$ Department of Physics, Kent State University, Kent, Ohio 44242, USA \\ ${ }^{3}$ Fundamentals of Advanced Materials Group, University of Delft, 2629 HS Delft, The Netherlands
}

(Received 16 June 2003; published 20 October 2003)

\begin{abstract}
Various optical and dynamic light scattering studies have been conducted on the nematic phase of an oxazole-derived bent-core liquid crystal. At optical length scales and in the absence of applied fields, homogeneously aligned samples of this material, which has an oxazole heterocyclic ring in the central core, are found to behave more like a conventional straight-core nematic than a previously investigated ester-based class of bent-core molecules that have a benzene ring as the core linkage between the two arms of the bow-shaped molecule. In particular, the nematic refractive indices of the oxazole compound combine in the standard way [i.e., $\sqrt{\left(2 n_{o}^{2}+n_{e}^{2}\right) / 3}$ ] to match the isotropic value throughout the nematic range, and the observed director fluctuation modes have relaxation rates comparable to those of the usual thermotropics. However, polarized light scattering data reveal evidence of weak biaxial fluctuations, and indications of electric-field-induced biaxiality are observed in the refractive index measurements.
\end{abstract}

DOI: 10.1103/PhysRevE.68.041704

\section{INTRODUCTION}

In the last several years studies of thermotropic liquid crystals of bent-core molecules have shown a number of phenomena [1-3], most notably the ferro- and antiferroelectricity and chirality in liquid crystals composed of achiral molecules. Although these materials tend to form only smectic and columnar phases [4], recently a number of materials were found that show nematic phases too [5-9]. So far experimental observations suggest a potentially wide range of behavior: uniaxial [5], uniaxial with biaxial fluctuations [9], and biaxial $[8,10]$, although the biaxiality has not yet been proven explicitly. Symmetry considerations revealed that the bow shape of the molecules can give rise to a number of distinct states of nematic ordering, including biaxial phases [11]. Obviously the opening angle $\psi$ between the mesogenic arms should play a major role in the formation of the actual phases. Recently, a molecular field theory [12] predicted that the biaxial nematic phase may appear for $\psi \sim 110^{\circ}$, whereas Monte Carlo simulations could account for only a uniaxial nematic phase when $\psi>135^{\circ}$ [13]. These experimental and theoretical results therefore point to the importance of detailed studies of the structure of the nematic phase of bentcore molecules.

Motivated by this we carried out detailed optical experiments on the nematic phase of a bent-core liquid crystal derived from 2,5-bis( $p$-hydroxyphenyl)-1,3-oxazole (and abbreviated 12-BPO); the general synthetic pathway is reported in Ref. [7]. The molecular structure and phase sequence of 12-BPO in bulk in cooling are given in Fig. 1. The molecule has a bent core with an opening angle estimated to be $140^{\circ}$ $\pm 5^{\circ}$. Recent $\mathrm{x}$-ray studies of this material suggest that the nematic phase in homogeneously aligned films appear to be biaxial [10]. Our studies, performed with similarly aligned samples, show that at optical length scales and in the absence of applied fields the material is optically uniaxial and that the evidence for biaxial fluctuations is rather weak, whereas strong indications of biaxiality are observed in refractive index measurements when an electric field is applied.
PACS number(s): 61.30.Eb, 61.30.Cz, 78.20.-e

\section{EXPERIMENTAL RESULTS AND DISCUSSION}

Polarizing microscopic observations, polarization current, $\mathrm{dc}$ resistivity and dynamic light scattering measurements were carried out on films of 12-BPO sandwiched between transparent indium tin oxide (ITO) electrodes in the thickness range between 2 and $20 \mu \mathrm{m}$ both with either homogenous planar or homeotropic alignment layers. In addition, 4-6 $\mu \mathrm{m}$ thick Fabry-Pérot cells were employed; the inner surfaces of these cells were coated with highly reflective ( $\sim 99.95 \%$ in the $1550 \mathrm{~nm}$ range) dielectric multilayers, then with ITO, and finally with homogeneous alignment layers.

Dynamic light scattering (DLS) measurements were carried out in standard optical cells with $10 \mu \mathrm{m}$ spacing between substrates that were treated for homogeneous director alignment. The sample was cycled through the transition to the isotropic phase, after which a uniform nematic alignment was observed across the cell in the polarizing microscope. The cell was contained in an oven with $2 \mathrm{mK}$ long-term
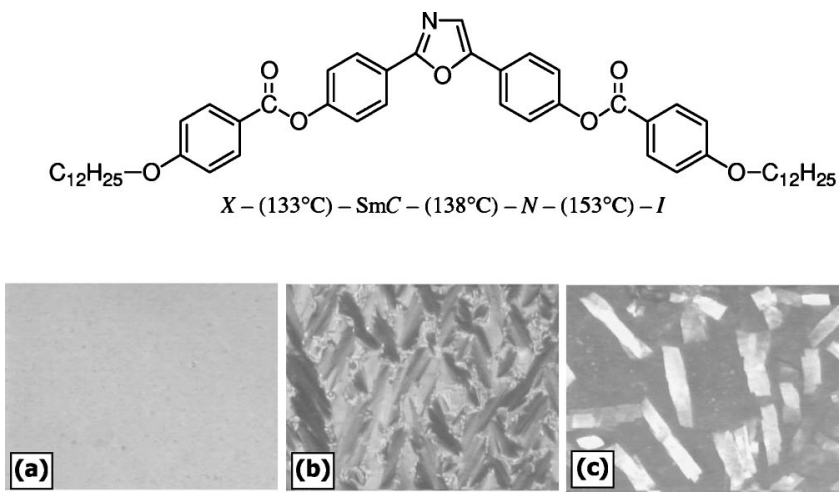

FIG. 1. Top: Molecular structure and phase sequence on cooling as measured in thick films of 12-BPO. Bottom: Corresponding textures observed at $E=0$ in a $10 \mu \mathrm{m}$ cell with planar anchoring: (a) $147^{\circ} \mathrm{C}$ : nematic phase; (b) $132{ }^{\circ} \mathrm{C}$ : smectic- $C$ phase; (c) $127^{\circ} \mathrm{C}$ : solid optically isotropic phase $(X)$ with crystal domains that formed after an hour. Pictures represent $0.4 \mathrm{~mm} \times 0.3 \mathrm{~mm}$ areas. Crossed polarizers are $45^{\circ}$ away from the rubbing direction. 

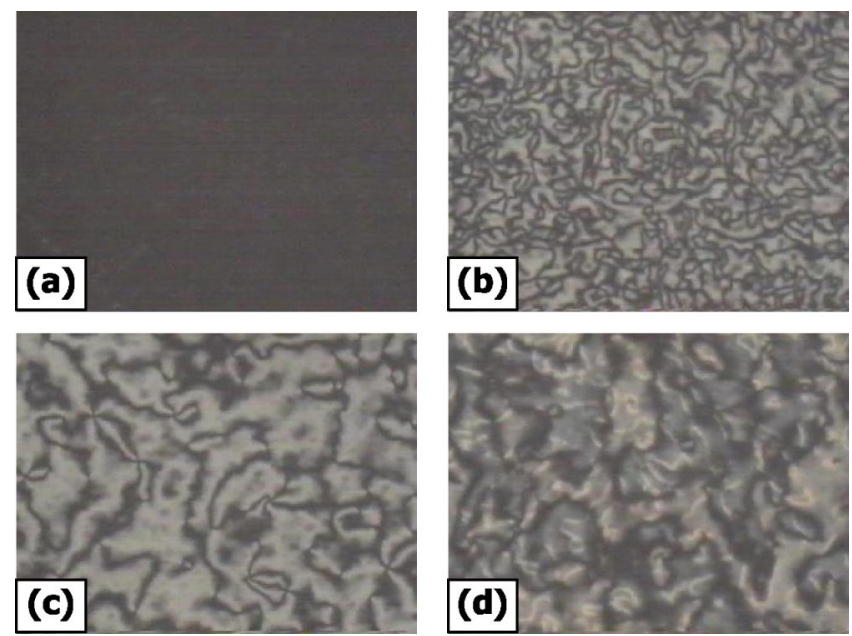

FIG. 2. Upper row $(0.4 \mathrm{~mm} \times 0.3 \mathrm{~mm}$ area): Textures of a $2 \mu \mathrm{m}$ cell of 12-BPO with homeotropic anchoring observed between crossed polarizers: (a) $142{ }^{\circ} \mathrm{C}$ at $E=0$; (b) $142^{\circ} \mathrm{C}$ at $E$ $=5 \mathrm{~V} / \mu \mathrm{m}, f=1 \mathrm{kHz}$. Lower row $(0.16 \mathrm{~mm} \times 0.12 \mathrm{~mm}$ area $)$ : Schlieren textures at $E=0$ : (c) $154{ }^{\circ} \mathrm{C}$; (d) $148{ }^{\circ} \mathrm{C}$.

temperature stability and optical access. The sample was illuminated with a focused $35 \mathrm{~mW}$ helium-neon laser (Spectra-Physics Model 127, $\lambda=633 \mathrm{~nm}$ ).

\section{Polarizing microscopy, refractive index, and electrical transport studies}

In films with planar anchoring the alignment is uniform, with the optical axis parallel to the rubbing direction. Under application of an electric field the extinction angle remains unchanged, whereas the birefringence slightly increases. The first order blue color indicates a birefringence of $\sim 0.1$ [see Fig. 1(a)].

The nematic phase on cooling exists between 153 and $138^{\circ} \mathrm{C}$. Between 138 and $133^{\circ} \mathrm{C}$ a $\mathrm{SmC}$ phase exists in thick $(d>6 \mu \mathrm{m})$ films [see Fig. 2(b)]. Below $133^{\circ} \mathrm{C}$ a solid, optically isotropic $X$ phase appears, which could not be switched by applied fields. Below $127^{\circ} \mathrm{C}$ the $X$ phase crystallizes out in a few hours [see Fig. 2(c)]. We note that electric current measurements under triangular voltages do not show any polarization in either the nematic or the smectic phase. In heating from the crystalline texture the material melts directly to the nematic phase at $146^{\circ} \mathrm{C}$, which then has a clearing point at $156^{\circ} \mathrm{C}$. In thinner $(d<6 \mu \mathrm{m})$ cells only one weakly birefringent solid phase appears below the nematic phase, indicating the metastable nature of the smectic phase.

In $2 \mu \mathrm{m}$ cells with homeotropic anchoring a quasiisotropic texture appears [see Fig. 2(a)], which can be reversibly switched above $142^{\circ} \mathrm{C}$ to a Schlieren texture [see Fig. 2(b)]. This clearly shows that the structure is uniaxial with negative dielectric anisotropy. On heating above $148^{\circ} \mathrm{C}$ it eventually becomes birefringent even at zero fields. In this case the electric field only slightly enhances the birefringence. On cooling from the isotropic phase in zero fields, first a Schlieren texture forms [see Fig. 2(c)], which contains four brush defects, indicating a tilted uniaxial structure. On
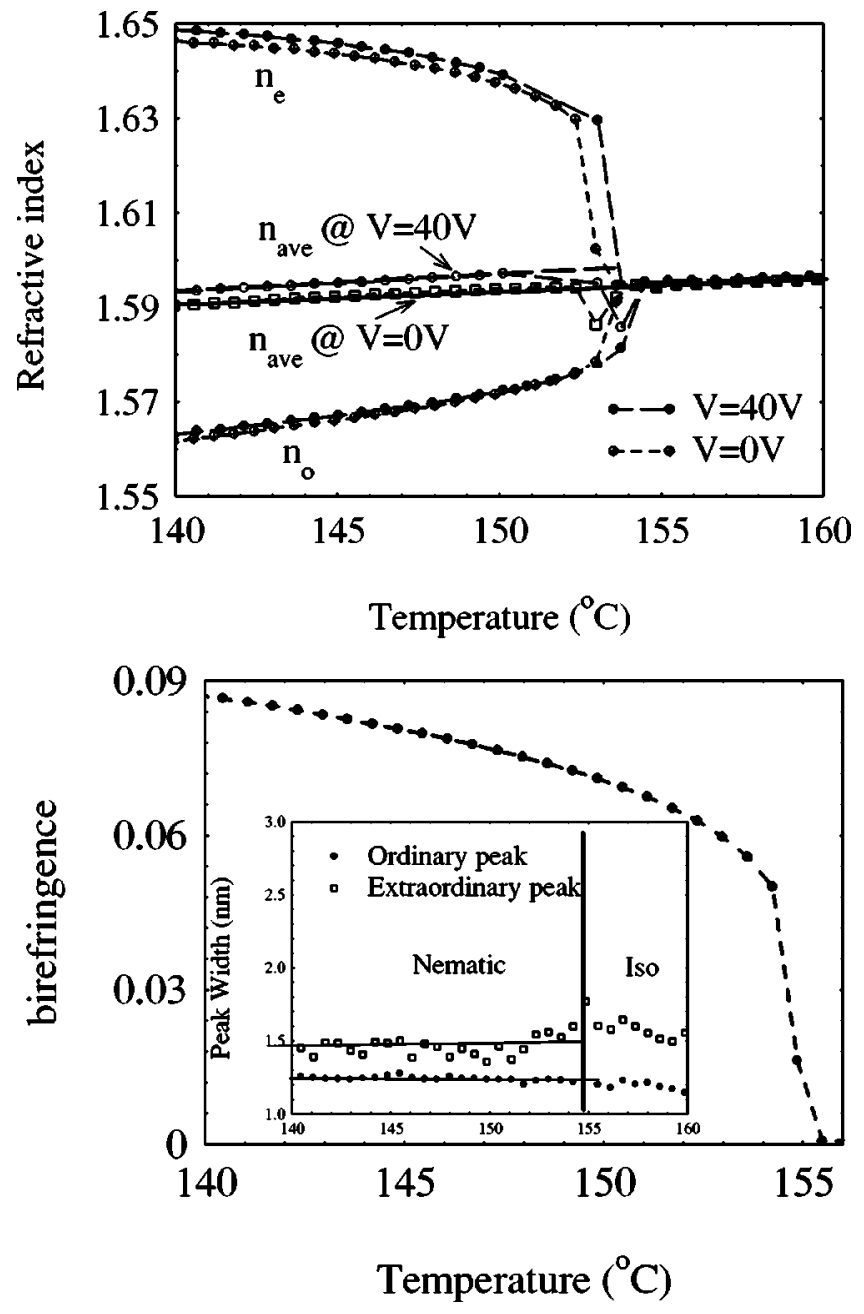

FIG. 3. Top: Temperature dependence of the refractive index of 12-BPO, as calculated from the Fabry-Pérot peaks, in zero applied electric field and in a field of $40 \mathrm{~V}$ across $5 \mu \mathrm{m}$. The average refractive index is calculated as $n_{\mathrm{av}}=\sqrt{\left(n_{e}^{2}+2 n_{o}^{2}\right) / 3}$. Bottom: Temperature dependence of the birefringence measured in the infrared range $(\lambda \sim 1.5 \mu \mathrm{m})$. Inset: Temperature dependence of the peak width for the extraordinary and ordinary rays.

further cooling the four brushes eventually disappear and simultaneously the overall texture becomes darker [see Fig. 2(d)]. Below about $145^{\circ} \mathrm{C}$ the texture between crossed polarizers becomes completely dark again and can be switched reversibly between Schlieren and black textures.

The refractive indices and the birefringences were measured in Fabry-Pérot cells at $\sim 1.5 \mu \mathrm{m}$ light wavelength. Here we note that the birefringence increases toward lower wavelengths (e.g., $\Delta n \sim 0.1$ at $0.45 \mu \mathrm{m}$ ) following the normal Cauchy formula [14]. In Fig. 3 (upper panel) we show the temperature dependence of the refractive indices as determined from the ordinary and extraordinary Fabry-Pérot peaks measured in zero field. We also show the average refractive index calculated as $n_{\mathrm{av}}=\sqrt{\left(2 n_{o}^{2}+n_{e}^{2}\right) / 3}$. It can be seen that in zero field $n_{\text {av }}$ basically corresponds to the isotropic value of the refractive index, indicating optical uniaxiality over the entire nematic range. On the other hand, when a sufficiently high field is applied (specifically $40 \mathrm{~V}_{\text {rms }}$ at 1 


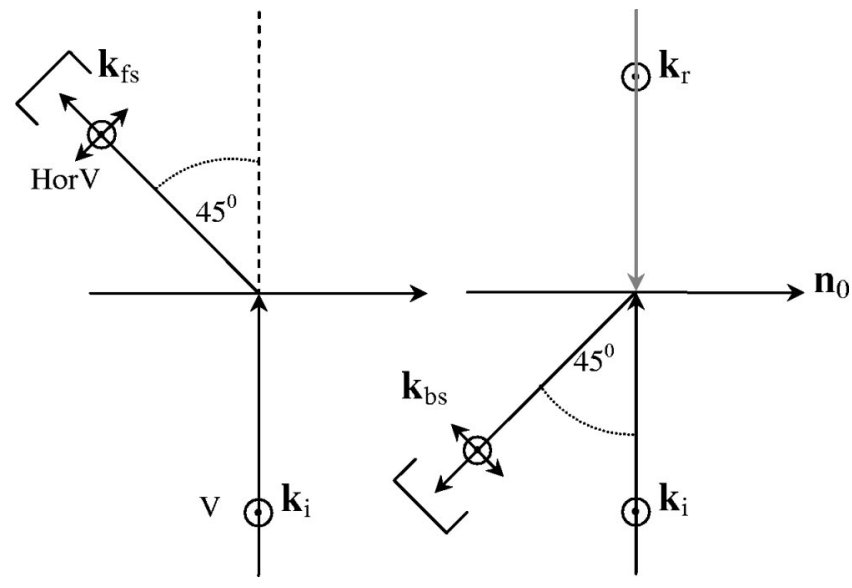

FIG. 4. Forward (left) and backward (right) light scattering geometries. The latter includes the contribution of an "effective" forward scattering process generated by back reflection of the main laser beam at the glass-air interface on the far side of the sample cell.

$\mathrm{kHz}$ across $5 \mu \mathrm{m}$ ), the figure shows that both the extraordinary and the ordinary indices are shifted to larger values, and the average refractive index has a jump at the transition to the isotropic phase $\left(n_{\mathrm{av}}-n_{\text {iso }}=0.004\right)$. Although the increase of the extraordinary index may be attributed to a small zerofield pretilt that is then suppressed in the presence of a field, the increase of the ordinary refractive index is a strong indication of a field-induced biaxiality [15].

The widths of the zero-field ordinary and extraordinary Fabry-Pérot peaks are shown in the inset to Fig. 3 (bottom). In addition to the parallelism and refractivity of the cell plates (which can be assumed to be temperature independent), only the director and nematic order parameter fluctuations and misalignments can influence the width of the peaks. The order parameter fluctuations affect both peaks, whereas azimuthal misalignment influences only the extraordinary component, and biaxial fluctuations impact only the ordinary peak. It is observed that the ordinary peak is basically temperature independent, indicating that the biaxial fluctuations are small over the entire nematic phase. This is in marked contrast to the significant biaxial fluctuations of an ester-based bent-core compound (abbreviated as 12-CPOB) [9], where an increase in the ordinary peak width was observed in the lower temperature range of the nematic phase.

\section{Dynamic light scattering}

We carried out dynamic light scattering measurements to search with greater sensitivity for evidence of biaxial fluctuations in the nematic phase of 12-BPO. The application of DLS was also motivated by recent results [9] obtained by us on the bent-core material 12-CPOB, where the effect of biaxial fluctuations was observed in the cross section for polarized scattering in the lower portion of the nematic phase and in the absence of any applied field.

The scattering geometries are shown schematically in Fig. 4. The director $\left(\vec{n}_{0} \equiv \hat{z}\right)$ was fixed in the scattering $(x-z)$ plane, with the incident (scattered) light polarization vertical (horizontal) to this plane. This geometry corresponds to de-

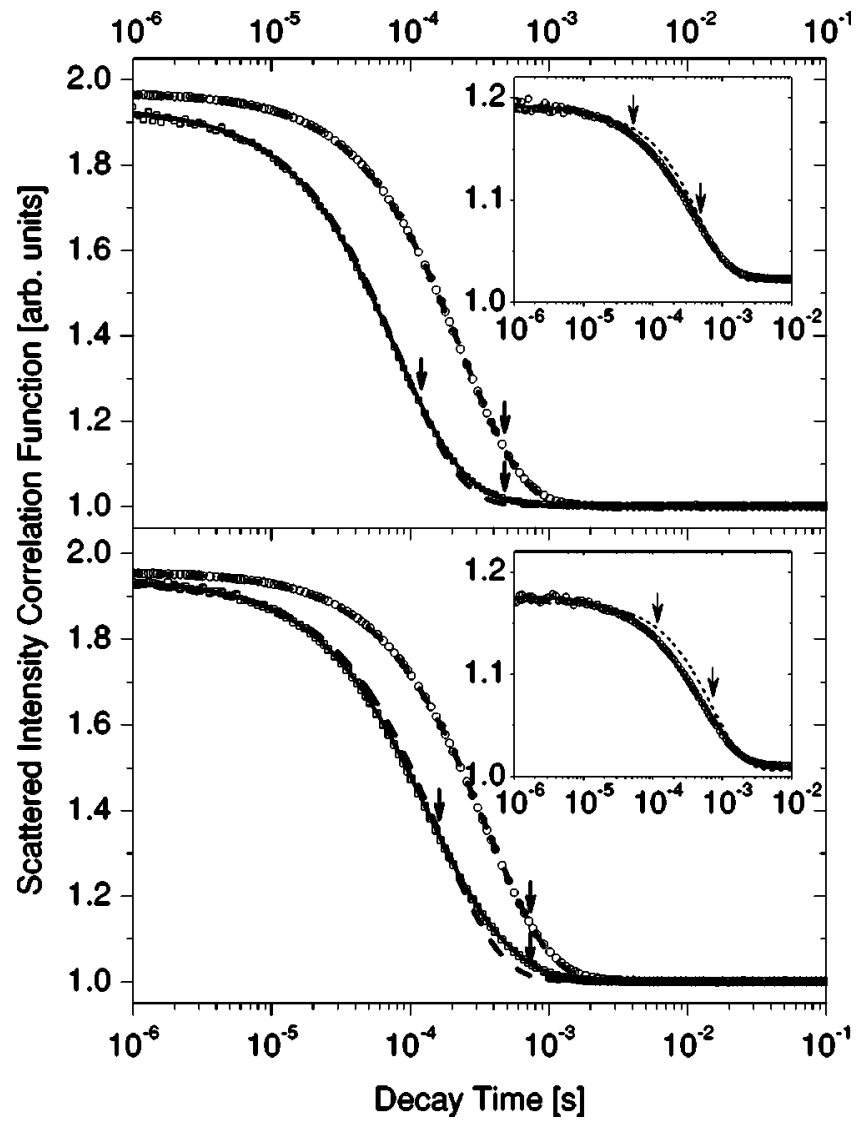

FIG. 5. Top and bottom panels show time correlation functions of the scattered intensity measured in the nematic $\left(146.12^{\circ} \mathrm{C}\right)$ and smectic- $C\left(135.74{ }^{\circ} \mathrm{C}\right)$ phases of $12-\mathrm{BPO}$. Open circles (squares) in the main figure correspond to data from forward (backward) $\mathrm{VH}$ scattering, and the insets show data from forward VV scattering. Dashed (solid) curves are fits to a single (constrained double) exponential decay. Downward arrows indicate the fitted relaxation times $(1 / \Gamma)$.

polarized $(\mathrm{VH})$ scattering and selects the twist-bend (tb) normal mode of the director fluctuations in a conventional nematic [16]. The relaxation rate of the tb mode is given by $\Gamma_{\mathrm{tb}}$ $=\left(K_{2} q_{x}^{2}+K_{3} q_{z}^{2}\right) / \eta_{\mathrm{tb}}$, where $K_{2}$ and $K_{3}$ are the twist and bend Frank elastic constants respectively, $\eta_{\mathrm{tb}}$ is an effective viscosity [16], and $\vec{q}=\left(q_{x}, q_{z}\right)$ is the scattering vector. The analyzer could be automatically rotated to the $V$ position, selecting a polarized $(V V)$ scattering process, and the incident and scattering angles ( $\theta_{i}$ and $\theta_{s}$, defined with respect to the cell normal $\hat{x}$ ) were also under computer control. The scattering vector $\vec{q}$ was calculated from the laboratory values of $\theta_{i}$ and $\theta_{s}$ plus the standard formulas for a uniaxial nematic liquid crystal [16].

Two limits of the tb mode were studied in detail: a forward scattering (FS) geometry with $\theta_{i}=0, \theta_{s}=45^{\circ}, q_{z}^{2} / q_{x}^{2}$ $=0.012, \Gamma_{\mathrm{tb}} \cong K_{3} q_{z}^{2} / \eta_{\mathrm{tb}}$, and a backward scattering (BS) geometry with $\theta_{i}=0, \theta_{s}=135^{\circ}, q_{z}^{2} / q_{x}^{2}=18, \Gamma_{\mathrm{tb}} \cong K_{2} q_{x}^{2} / \eta_{\mathrm{tb}}$. (These limits for $\Gamma_{\mathrm{tb}}$ assume that $K_{2}$ and $K_{3}$ are of the same order of magnitude, which is typically the case in thermotropics and consistent with the results described below.)

Figure 5 displays typical data for the time correlation 
functions of the scattered intensity, which are plotted over the relevant range in time where the decay of the correlations is observed. The top panel shows typical results for the nematic phase (at a temperature of $146.12{ }^{\circ} \mathrm{C}$ ) obtained for the $\mathrm{VH}$ scattering process in the FS and BS geometries (circles and squares, respectively, in the main figure) and for the VV process in the FS geometry (inset). The bottom panel shows similar data obtained in the smectic- $C$ phase (at $135.74{ }^{\circ} \mathrm{C}$ ). The dashed lines for $\mathrm{VH}$ forward scattering are fits to a single overdamped mode, from which a value of $2 \Gamma_{\mathrm{tb}}$ is extracted (corresponding to the homodyne limit for the intensity correlations). The quality of the fits is excellent. On the other hand, the measured correlation functions for $\mathrm{VH}$ backscattering are not accurately described by a single mode, as shown by the systematic deviation of the data from the dashed line near the tail of the decay. The reason for this deviation is that tb fluctuations for two different scattering vectors contribute in the BS geometry. The dominant contribution comes from the mode with large $q_{z}$ and small $q_{x}$ set by the backward geometry. However, we can also expect a weak contribution due to scattering of the laser light reflected back into the sample from the planar air-glass interface at the outer cell surface. As indicated in Fig. 4, this scattering is equivalent to a forward process with $\theta_{i}=0, \theta_{s}=45^{\circ}$. Even though the reflected laser intensity is only $\sim 4 \%$ of the incident intensity, it can still produce significant scattering because of the hydrodynamic $q$ dependence of the fluctuations, i.e., $\Gamma_{\mathrm{tb}} \sim q^{2}$. For the "effective" forward scattering from the reflected beam, $q^{2}=50.0 \mu \mathrm{m}^{-2}$, while for the pure backscattering component $q^{2}=950 \mu \mathrm{m}^{-2}$. Since the scattering amplitude scales as $q^{-2}$, we see that secondary scattering produced from even weakly reflected light can be significant in the BS geometry. However, our choice of identical acute detector angles (with respect to the incident beam and cell surface normal) in the FS and BS geometries allows us to fix the relaxation rate of the "effective" forward scattering component detected in the BS geometry signal to the value measured separately in the FS geometry. This greatly improves the precision of $\Gamma_{\mathrm{tb}}$ for the pure BS process that can be extracted from a double exponential fit to the correlation data. As demonstrated in Fig. 5 by the solid lines, the constrained double exponential fits to the BS data are excellent. We verified that the "contamination" from scattering of the reflected main beam gets much worse as the detector is moved closer to the direct backscattering direction (i.e., as $q^{2}$ for the "effective" forward scattering process is sharply decreased). The selection of $45^{\circ}$ for the angles with respect to normal incidence represents a compromise between the severe contamination that would occur in a BS geometry with $q_{z}=0$ and the increasing contribution of the bend elastic constant that comes from making $q_{z}$ larger.

Let us now consider the results for VV scattering obtained in the FS geometry. For a uniaxial nematic with the director oriented in the scattering plane, the cross section for VV scattering due to director fluctuations vanishes, and the correlation function amplitude is therefore predicted to be zero. In practice, one always expects a weak signal due to alignment mosaic. Such an effect is indeed observed in the correlation functions presented in the insets to Fig. 5. To analyze
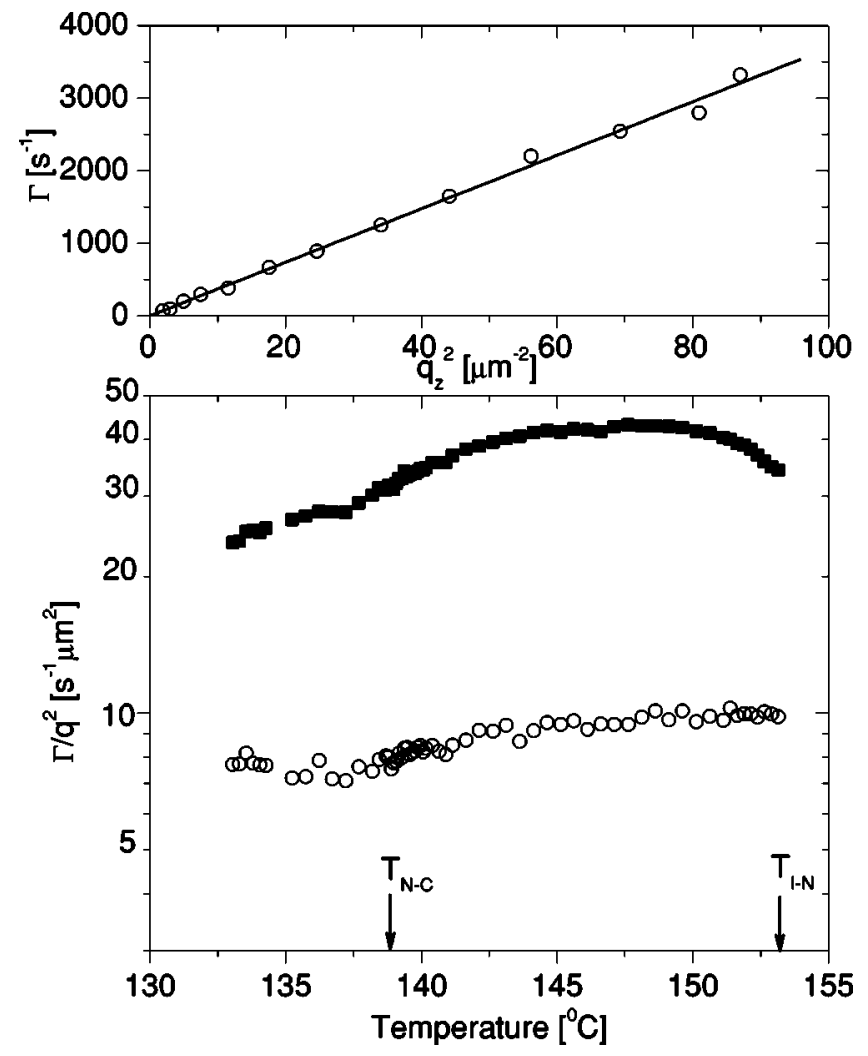

FIG. 6. Top panel: Expected hydrodynamic $q$ dependence of the relaxation rate $\Gamma$ of the nematic director fluctuations measured in 12-BPO. Bottom: Elastic constant to viscosity ratios $\Gamma / q^{2}=K / \eta$ obtained in the nematic and smectic- $C$ phases. The squares (circles) correspond to primarily bend (twist) fluctuations.

these data, we first tried single mode fits (in the heterodyne limit) with the relaxation rates fixed to the values obtained for the tb director mode from the corresponding $\mathrm{VH}$ scattering data taken under the exact same conditions. These fits are shown as dashed lines in the insets, and are slightly off the data. Apparently a second mode may contribute to the VV scattering. The solid lines show double exponential fits with the relaxation rates of the slower mode constrained as described; for the faster mode, we obtain values of $\Gamma$ $=17800 \mathrm{~s}^{-1}$ and $7900 \mathrm{~s}^{-1}$ in the nematic and smectic- $C$ phases, respectively. As argued in our previous study of $12-$ CPOB [9], the faster mode observed for the VV process is attributable to amplitude fluctuations of the biaxial order parameter. However, in the case of $12-\mathrm{CPOB}$, this mode was stronger-the ratio of its amplitude to the amplitude of the director mode approached 0.5 near the transition to the smectic- $C$ phase. In the present case, the ratio is approximately three times smaller. We should point out that our VH forward scattering geometry would also be sensitive to orientational fluctuations of the biaxial order parameter for both potential orientations of the biaxial "director" $\vec{m}$ (i.e., $\vec{m}$ perpendicular to $\vec{n}$ and parallel or perpendicular to the substrate normal). As Fig. 5 indicates, we find no evidence of a distinct contribution to the $\mathrm{VH}$ correlation function due to $\vec{m}$, which is entirely consistent with the absence of detectable large-scale biaxial order deduced from the behavior of the refractive indices in Fig. 3. 


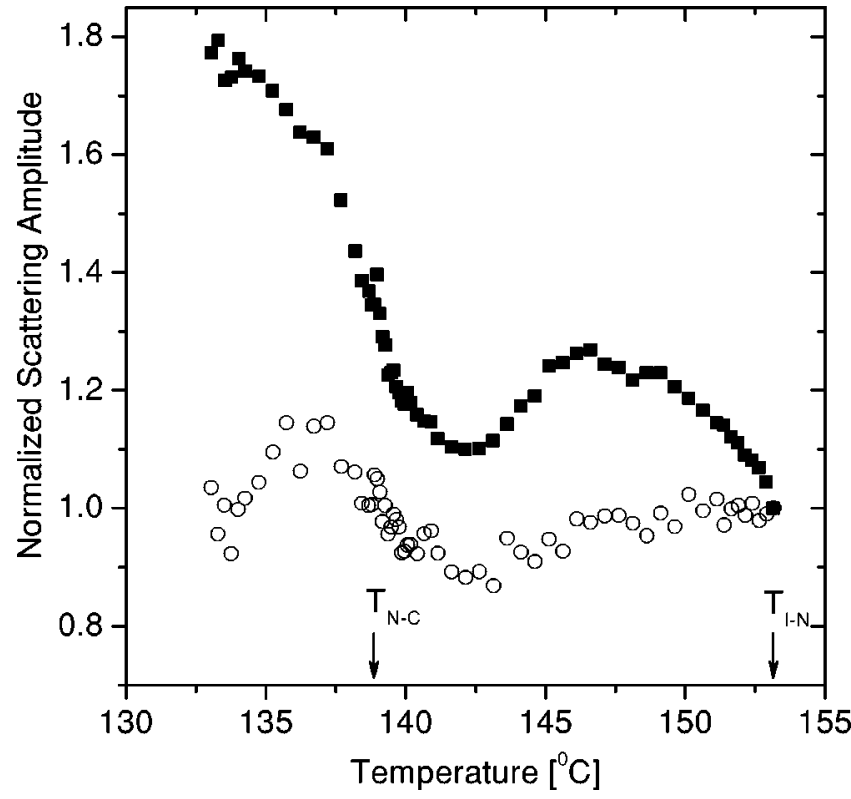

FIG. 7. Temperature dependence of the scattering amplitudes for predominantly bend (squares) and twist (circles) fluctuations in the nematic and smectic- $C$ phases of 12-BPO.

The top panel of Fig. 6 demonstrates the expected hydrodynamic $q$ dependence of the tb director mode, measured in the FS geometry with $q_{z}^{2} \gg q_{x}^{2}$. In the bottom panel, we plot the temperature dependence (on cooling) of the viscoelastic ratios $\Gamma_{\mathrm{tb}} / q_{z}^{2} \cong K_{3} / \eta_{\mathrm{tb}}$ and $\Gamma_{\mathrm{tb}} / q_{x}^{2} \cong K_{2} / \eta_{\mathrm{tb}}$ obtained from the analysis of the correlation functions in the FS and BS geometries, respectively, and according to the procedure described above. The twist-dominated viscoelastic ratio is approximately three times smaller than the bend ratio, which is rather characteristic of thermotropic nematics. The magnitude of the viscoelastic ratio for bend is $K_{3} / \eta_{\text {tb }}$ $\simeq 40 \mu \mathrm{m}^{2} \mathrm{~s}^{-1}$, or about five times lower than for typical straight core nematics, although it is considerably more normal than the value $K_{3} / \eta_{\mathrm{tb}} \simeq 1.5 \mu \mathrm{m}^{2} \mathrm{~s}^{-1}$ previously observed by us in the ester derivative 12-CPOB [9]. The temperature dependence of the viscoelastic ratios in 12-BPO is unremarkable through the nematic range and across the transition to the smectic- $C$ phase; in particular, we observe no significant pretransitional behavior. The corresponding scattering amplitudes (see Fig. 7), which are proportional to the ratio of the dielectric anisotropy to the elastic constants, also fail to reveal any dramatic temperature dependence.

\section{DISCUSSION}

In several respects the optical and fluctuation properties of the nematic phase of the bent-core compound 12-BPO are similar to conventional straight-core (calamitic) nematics. In zero field, the optical anisotropy exhibits the usual characteristics of a uniaxial system. In addition the dominant fluctuations are hydrodynamic director modes with the relaxation rate for bend being several times that for twist (and fairly comparable to the rate observed at optical wave vectors in straight-core nematics). The similarities observed by us between the nematic phase of 12-BPO and typical calamitic nematics, and the differences we noted relative to the esterbased 12-CPOB, may correlate with the opening angle $\psi$ between the two arms of the bow: in a typical calamitic $\psi$ $\sim 180^{\circ}$; in 12 -BPO, $\psi>140^{\circ}$, whereas in 12 -CPOB $\psi$ $\sim 120^{\circ}$. For $\psi>135^{\circ}$ the arguments of Ref. [12] and the Monte Carlo simulation results of Lansac et al. [13] do not favor spontaneous biaxiality. Of course, one can perhaps still imagine that the opening angles of the bows may be smaller in close packed structures than is estimated in the gas state. In this case, as we previously suggested [9], temporary formation of microscopic smecticlike clusters may occur. The local packing of such clusters potentially hinders rotations about the axis connecting the two ends of the bent-core molecules and consequently enhances the cross section for biaxial fluctuations. On the other hand, such clusters should enhance the orientational and translational viscosities, which were indeed observed in 12-CPOB, but not in 12-BPO. In any case, the combined results of the present and our previous studies reveal that bent-core nematics exhibit interesting variations in their optical and viscoelastic properties that are apparently correlated to a change in $\psi$.

The temperature dependence of the relaxation rates and elastic constants is weak within the bulk of the nematic phase, with no anomalous pretransitional behavior at the transition to the smectic- $C$ phase in either bend or twist viscoelastic parameters. The absence or suppression of critical temperature dependence in these parameters (particularly for bend fluctuations) at the nematic to smectic- $C$ transition has not to our knowledge been adequately explained theoretically, although it was previously observed in certain straightcore thermotropic mixtures [16]. The Chen-Lubensky theory $[17,18]$ of the nematic to smectic- $C$ transition predicts an essentially linear decrease in scattered intensity for bend, $I$ $\sim T-T^{*}$, with $T^{*}$ an apparent critical temperature. However, the straight-core mixtures studied in Ref. [16] actually showed a slight increase in $I$ within $1{ }^{\circ} \mathrm{C}$ of the transition, which is somewhat similar to what we observe in Fig. 7.

Although the evidence of biaxial fluctuations in the zerofield state of 12-BPO is rather weak, refractive index measurements provide significant indications of field-induced biaxiality. This behavior certainly merits more detailed study. Moreover, our study is not sensitive to biaxial correlations at suboptical length scales, and the possibility of significant shorter-range biaxial structure (in the absence of bulk fields) should be investigated by appropriate techniques.

\section{ACKNOWLEDGEMENTS}

Two of us (S.S. and S.S.) gratefully acknowledge support by the NSF under Grant No. DMR-9904321. A.O. and A.J. acknowledge support from CoAdna, Inc. and helpful discussions with Professor J. Kelly. We are grateful to S. Kumar and E. Samulski for a stimulating conversation. 
[1] T. Niori, T. Sekine, J. Watanabe, T. Furukawa, and H. Takezoe, J. Mater. Chem. 6, 1231 (1996).

[2] D.R. Link, G. Natale, R. Shao, J.E. Maclennan, N.A. Clark, E. Korblova, and D.M. Walba, Science 278, 1924 (1997).

[3] G. Heppke, A. Jákli, S. Rauch, and H. Sawade, Phys. Rev. E 60, 5575 (1999).

[4] G. Pelzl, S. Diele, and W. Weissflog, Adv. Mater. (Weinheim, Ger.) 11, 707 (1999).

[5] J. Matraszek, J. Mieczkowski, J. Szydlowska, and E. Gorecka, Liq. Cryst. 27, 429 (2000); I. Wirth, S. Diele, A. Eremin, G. Pelzl, S. Grande, L. Kovalenko, N. Pancenko, and W. Weissflog, J. Mater. Chem. 11, 1642 (2001); W. Weissflog, H. Nádasi, U. Dunemann, G. Pelzl, S. Diele, A. Eremin, and H. Kresse, ibid. 11, 2748 (2001).

[6] E. Mátyus and K. Keserü, J. Mol. Struct. 543, 89 (2001).

[7] T.J. Dingemans and E.T. Samulski, Liq. Cryst. 27, 131 (2000).

[8] D. Shen, S. Diele, G. Pelzl, I. Wirth, and C. Tschirske, J. Mater. Chem. 9, 661 (1999).
[9] S. Stojadinovic, A. Adorjan, S. Sprunt, H. Sawade, and A. Jákli, Phys. Rev. E 66, 060701(R) (2002).

[10] B. Acharya, A. Primak, T. Dingemans, E.T. Samulski, and S. Kumar (unpublished).

[11] T.C. Lubensky and L. Radzihovsky, Phys. Rev. E 66, 031704 (2002).

[12] G.R. Luckhurst, Thin Solid Films 393, 42 (2001).

[13] Y. Lansac, P.K. Maiti, N.A. Clark, and M.A. Glaser, Phys. Rev. E 67, 011703 (2003).

[14] L. Cauchy, Bull. Des. Sci. Math. 14, 9 (1830).

[15] D.A. Dunmur, K. Szumilin, and T.F. Waterworth, Mol. Cryst. Liq. Cryst. 149, 385 (1987).

[16] P.G. de Gennes and J. Prost, The Physics of Liquid Crystals, 2nd ed. (Clarendon Press, Oxford, 1993), Chap. 3.

[17] L. Solomon and J.D. Litster, Phys. Rev. Lett. 56, 2268 (1986); J. Huang and J.T. Ho, Phys. Rev. A 38, 400 (1988).

[18] J. Chen and T.C. Lubensky, Phys. Rev. A 14, 1202 (1976). 